\title{
Insights into the interplay of latent toxoplasmosis, testosterone, cortisol and oxidative stress in screened schizophrenic patients in Egypt
}

\author{
Original \\ Naglaa SM El-Gebaly ${ }^{1}$, Magda SA Abd-Eltawab ${ }^{1}$, Alshaimaa MR Hamed ${ }^{1}$, \\ Article \\ Noha A Mahfouz ${ }^{2}$, Reham Abdelmaksoud ${ }^{2}$, Shadan Adel $^{2}$, Amal M Mahfoz ${ }^{3}$, \\ Mohammed K Rehan ${ }^{4}$, Eman H Elsebaei ${ }^{5}$
}

Departments of Medical Parasitology ${ }^{1}$, Psychiatric disorders and addiction ${ }^{2}$, Pharmacology
Internal Medicine
In $^{4}$, and Community Medicine
, Faculties of Medicine

\begin{abstract}
Background: Toxoplasma gondii is a wide-spread infection. Most cases are asymptomatic latent toxoplasmosis and its link with schizophrenia is of wide interest. Toxoplasmosis induces immunopathological and neuroendocrine alterations that induce activation of hypothalamic pituitary-adrenal axis resulting in higher glucocorticoid and testosterone levels. Oxidative stress also has a role since antioxidants as glutathione (GSH) were found to be reduced. Objective: To assess seroprevalence/serointensity of toxoplasmosis in schizophrenic patients in relation to the levels of testosterone, cortisol and GSH activity in order to evaluate these factors in the etiopathogenesis of schizophrenic patients harboring latent toxoplasmosis.

Subjects and Methods: In this cross sectional case control study, 120 schizophrenic inpatients were compared with 120 individuals attending the outpatients' clinics in the same age range, and after exclusion of schizophrenia. Schizophrenia was confirmed or excluded using positive and negative syndrome scale (PANSS). Levels of Toxoplasma IgG, free testosterone, cortisol and GSH were determined in both groups.

Results: Statistical analysis revealed no differences between cases and controls as regard age, gender, contact with pets, and Toxoplasma IgG seroprevalence. Toxoplasma IgG seroprevalence was higher in males compared to females and in rural areas than in urban areas. In Toxoplasma-positive patients, testosterone was higher in both genders and glutathione was lower, while no significant difference was documented in relation to PANSS, treatment with electroconvulsive-therapy (ECT) or cortisol level. Schizophrenic patients showed higher Toxoplasma antibody titer, cortisol, free testosterone levels in both genders and lower GSH than control. Male schizophrenic patients particularly showed lower GSH level and higher Toxoplasma antibody titer. Free testosterone level was significantly higher in male patients who had received ECT.

Conclusion: Latent toxoplasmosis may be involved in the etiology of schizophrenia. Screening and treatment of toxoplasmosis is advisable to prevent schizophrenia. Also, screening of schizophrenic patients for chronic toxoplasmosis and the fore mentioned parameters is recommended for prediction of complications and presents a promising approach for prevention and management.
\end{abstract}

Keywords: cortisol, glutathione, latent toxoplasmosis, schizophrenia, testosterone.

Received: 12 April 2019, Accepted: 5 May, 2019.

Corresponding Author: Naglaa SM El-Gebaly; Tel.: 002/01018248768, E-mail: nssoliman@kasralainy.edu.eg

Print ISSN: 1687-7942, Online ISSN: 2090-2646, Vol. 12, No. 2, Ausgust, 2019.

\section{INTRODUCTION}

Toxoplasma gondii, an obligate intracellular and opportunistic parasite, is one of the most widely spread infections all over the globe, affecting about $30 \%$ of the world population ${ }^{[1]}$. Unlike many other parasitic disorders, its distribution is not merely restricted to developing countries, but it also afflicts developed nations such as France, which is known for having a high incidence of toxoplasmosis ${ }^{[2]}$. The wide range of this infection is attributed to the multiplicity of its modes of infection. Besides, being a zoonosis, T. gondii oocysts shed by the feline definitive host can be acquired by the mammalian intermediate host through the ingestion of contaminated food and water. Also, improperly cooked meat containing tissue cysts constitute a potential source of infection. Additional modes of infection include organ transplantation, blood transfusion and congenital transmission from mother to fetus ${ }^{[3]}$.

The clinical range includes acute toxoplasmosis presenting with a variety of nonspecific symptoms such as sore throat and lymphadenitis, opportunistic 
toxoplasmosis in the immunocompromised and congenital toxoplasmosis especially known for its ocular and neurological manifestations ${ }^{[4]}$. Even though infection is common, clinical disease is manifested to a much lesser extent, where most cases are asymptomatic, a condition called latent toxoplasmosis ${ }^{[5]}$. Although patients that are harboring latent toxoplasmosis are asymptomatic, it was found to be incriminated in the development of mental retardation in children ${ }^{[6]}$. In addition to the mentioned presentations of toxoplasmosis, recent suggestions implicate $T$. gondii in other disorders of undefined etiology. Of special interest is its reported link to a group of neuropsychiatric disorders such as autism, bipolar disorder, schizophrenia and degenerative diseases such as Alzheimer's and Parkinson's diseases ${ }^{[7]}$. Association with these clinical conditions was motivated by the multiple effects of T. gondii on host physiology. Besides, the resulting immunopathological changes, T. gondii is also known to induce neuroendocrine alterations ${ }^{[8,9]}$.

Most patients with schizophrenia suffer from a lifetime of disability and about $10 \%$ commit suicide $\left.{ }^{[10}\right]$. The etiology of schizophrenia is thought to be due to several factors. In family studies, genetic factors were found to play a crucial role ${ }^{[11]}$, whereas epidemiological researches indicated that environmental exposures, such as winter-spring births, peri- and postnatal infections and urban births are risk factors for the development of schizophrenia later in life ${ }^{[12]}$. Infectious agents, including T. gondii, were considered relevant in triggering schizophrenia ${ }^{[13]}$. Toxoplasmosis has been linked to schizophrenia through epidemiological data, serological evidence, neuropathological and molecular studies ${ }^{[14]}$. The causal relationship was speculated to involve susceptibility genes targeting, pro-inflammatory cytokines, neurotransmitters and hormones ${ }^{[15]}$. It was explained that $T$. gondii encysted in the host tissues constitutes a source of chronic infection, which leads to the activation of hypothalamic pituitary adrenal axis and subsequently, an increase in glucocorticoid concentration. This increase, in turn, is linked to neurosynaptic degeneration ${ }^{[9]}$.

Another hormonal change that may explain the link between toxoplasmosis and schizophrenia is the elevation of serum testosterone, which was reported in several studies in Toxoplasma seropositive patients ${ }^{[5,16,17]}$. Alterations of serum testosterone levels have also been found to affect the severity of clinical disease in schizophrenic patients ${ }^{[18]}$. In addition to neuroendocrine alterations, oxidative stress is one of the mechanisms of pathology that is shared by both schizophrenia and toxoplasmosis. Oxidative stress can be assessed by several markers including malonedialdehyde (MDA), which reflects the degree of lipid peroxidation, and antioxidants such as GSH. MDA was found to be increased in Toxoplasma seropositive patients, while GSH was found to be reduced ${ }^{[19]}$. In parallel, psychotic patients suffering from schizophrenia and bipolar disorder were shown to have a reduced serum level of glutathione ${ }^{[20]}$.

To our knowledge, implication of the previously mentioned factors in the pathogenesis of toxoplasmosis and schizophrenia have been studied separately. The causative relation between endocrine changes and oxidative status and these two diseases remain underinvestigated. The current study aims at assessing the seroprevalence/serointensity of toxoplasmosis in schizophrenic patients in relation to the levels of testosterone, cortisol and GSH activity in order to evaluate these factors in schizophrenic patients harboring latent toxoplasmosis.

\section{SUBJECTS AND METHODS}

This is a cross sectional case control study performed during the period from January 2018 to November 20 at Kasr-Al-Ainy Psychiatry and Addiction Hospital and Medical Parasitology Department, Faculty of Medicine, Cairo University, Cairo.

Subjects: A total number of 240 individuals divided into two equal groups were included in the study. Group 1 included inpatients (case group) ranging in age from 16 to 55 years; while group 2 included individuals attending the outpatients' clinics in the same age range, and after exclusion of schizophrenia. A data collection sheet covering sociodemographic and clinical information was fulfilled before serological testing. Non-cooperative patients, patients with other severe co-morbid disease or history of treatment of toxoplasmosis before sampling were excluded.

Study design: For psychiatric assessment of case group, diagnosis was confirmed by a structured clinical interview using the statistical manual of mental disorders (DSM) IV axis I disorder (SCID-I ${ }^{[21]}$. Psychotic symptom severity was assessed according to PANSS. A general psychopathology scale was also performed. The validity and reliability of the total syndrome (TS) scale (the sum of positive, negative and general scales) was established ${ }^{[22]}$. ECT was included as a severity marker since it is only indicated for severe cases. Blood samples were collected from both groups for evaluation of the seroprevalence of anti-Toxoplasma IgG, and determination of levels of free testosterone, cortisol and GSH.

Specimen collection and preparation: Three $\mathrm{ml}$ venous blood samples obtained by venipuncture were centrifuged at $3000 \mathrm{rpm}$ for $5 \mathrm{~min}^{[23]}$ at the Department of Parasitology, Kasr-Al-Ainy School of Medicine and separated sera were frozen until use. Repeated freezing and thawing or heat inactivation were avoided. Insufficient samples or those with incomplete information in the data collection sheet, with hemolysis, turbid or contaminated serum samples, were excluded. 
Parameters: ELISA was performed according to the manufacturer's instructions as described for Toxoplasma IgG ELISA (Calbiotech Inc, Beta Lab, Cairo, Egypt). For free testosterone and cortisol, ELISA was performed as described by Diametra Inc., and Bioactivia Diagnostic, respectively; both supplied by El-Mottahedeh Inc, Cairo, Egypt. Competitive immunoenzymatic colorimetric method was used for quantitative determination of free testosterone concentration. The cortisol kit used is a solid phase competitive ELISA, in which the intensity of color was considered inversely proportional to the cortisol concentration. Colorimetric method was performed as described for GSH (Bioactivia Diagnostic, ElMottahedeh Inc, Cairo, Egypt). The method is based on the reduction of 5,5' Dithiobis (2-nitrobenzoic acid) by GSH producing a yellow compound. According to the instruction manual, the reduced chromogen was considered directly proportional to GSH concentration and its absorbance was measured at $405 \mathrm{~nm}$.

Statistical analysis: Microsoft excel 2013 was used for data entry and the statistical package for social science (SPSS version 24) was used for data analysis. Simple descriptive statistics (arithmetic mean and standard deviation) were used for analysis of normal quantitative data using paired t test, one-way ANOVA and post-hoc tests. Frequencies were used for qualitative data and statistically analyzed by Chi-square and Fisher's exact tests where appropriate. The level of significance was set at probability $(P)$ value $<0.05^{[24]}$.

Ethical considerations: Both schizophrenic and nonschizophrenic patients were properly informed of the purpose of the study and were provided with consent forms. The study was performed in accordance to the ethical guidelines of the Faculty of Medicine, Cairo University; in addition to the approval of the Inpatient Psychiatry Hospital ethical committee.

\section{RESULTS}

All 120 schizophrenic patients and 120 nonschizophrenic control samples were subjected to measurement of Toxoplasma IgG, cortisol, free testosterone and GSH levels. Table (1) describes data of patients as regards duration of illness, different scales done to assess their schizophrenic illness and history of ECT to assess severity; besides past and family history of psychotic and mood disorders.

No significant difference was recorded between cases group and control group as regard age, gender, occupation, contact with pets, and Toxoplasma IgG seroprevalence $(P<0.05)$. There were significant higher Toxoplasma titers $(P=0.049)$, free testosterone levels in both genders $(P<0.001$ and 0.005 in males and females, respectively) and cortisol levels $(P<0.001)$ in both groups; while GSH level was significantly lower in cases group than control group $(P<0.001)$ (Table 2$)$.

In tables (3 and 4), Toxoplasma prevalence in schizophrenic cases showed significantly higher rate in male patients than in females $(P=0.03)$, and in rural than in urban areas $(P=0.002)$; while it was significantly lower in patients with history of drug abuse $(P=0.005)$ and patients with positive family history of psychotic disorders $(P=0.011)$. Prevalence was not higher in patients with history of contact with cats. There was no significant difference in patients with positive family history of mood disorders; and there were no significant differences related to positive syndrome scale (PS), negative syndrome scale (NS), general syndrome scale (GS), TS, treatment with ECT or cortisol level. In Toxoplasma positive patients; free testosterone levels in both genders were significantly higher $(P<0.001)$ while GSH was significantly lower $(P=0.045)$. Gender specification showed no significant difference in PS, GS and cortisol level (Table 4).

Table 1. Mean \pm SD of duration of illness, PANSS and history of schizophrenic cases.

\begin{tabular}{lcc}
\hline Relevant data & & Mean \pm SD \\
\hline Duration of illness (Years) & & $5 \pm 4$ \\
PS (7-49) & & $22 \pm 8$ \\
NS (7-49) & & $19 \pm 10$ \\
GS (16-112) & & $26 \pm 6$ \\
TS & Negative & $67 \pm 15$ \\
\hline Relevant data & Positive & No. (\%) \\
\hline Electro convulsive therapy & Negative & $48(40.0)$ \\
& Positive & $72(60.0)$ \\
Past history of psychosis & Negative & $120(100.0)$ \\
& Positive & $0(0.0)$ \\
Past history of medical disorders & Negative & $120(100.0)$ \\
Past history of drug abuse & Positive & $0(0.0)$ \\
& Negative & $114(95.0)$ \\
Family history of psychosis & Positive & $6(5.0)$ \\
& Negative & $87(72.5)$ \\
Family history of mood disorders & Positive & $33(27.5)$ \\
& & $99(82.5)$ \\
\hline \hline
\end{tabular}


Table 2. Comparison of sociodemographic data, Toxoplasma prevalence, mean \pm SD of Toxoplasma titers, cortisol, free testosterone and GSH levels among cases and control.

\begin{tabular}{|c|c|c|c|c|}
\hline & & No. of cases (\%) & No. of control (\%) & $P$ value \\
\hline \multirow[t]{2}{*}{ Gender } & Male & $81(67.5)$ & $88(73.3)$ & \\
\hline & Female & 39 (32.5) & $32(26.7)$ & 0.322 \\
\hline \multirow[t]{2}{*}{ Occupation } & Negative & $84(70.0)$ & $80(66.7)$ & \\
\hline & Positive & $36(30.0)$ & $40(33.3)$ & 0.578 \\
\hline \multirow{2}{*}{ Contact with pets } & Negative & $99(82.5)$ & $97(80.8)$ & \\
\hline & Positive & $21(17.5)$ & $23(19.2)$ & 0.739 \\
\hline \multirow[t]{4}{*}{ Toxoplasma titers } & Negative & $66(55.0)$ & $58(48.3)$ & \\
\hline & Positive & $54(45.0)$ & $59(49.2)$ & 0.154 \\
\hline & Borderline & $0(0.0)$ & $3(2.5)$ & \\
\hline & & No. $($ Mean \pm SD) & No. $($ Mean \pm SD) & $P$ value \\
\hline Age & & $120(30.23 \pm 8.252)$ & $120(31.5 \pm 8.750)$ & 0.248 \\
\hline Toxорlasma titers & & $120(1.43 \pm 1.217)$ & $120(1.129 \pm 1.129)$ & $0.049 *$ \\
\hline Cortisol & & $120(176.972 \pm 130.522)$ & $120(98.39 \pm 46.255)$ & $<0.001^{*}$ \\
\hline \multirow{2}{*}{ Free testosterone } & Male & $81(17.968 \pm 10.481)$ & $88(9.014 \pm 6.64)$ & $<0.005^{*}$ \\
\hline & Female & $39(6.5 \pm 8.52)$ & $32(2.37 \pm 3.53)$ & $<0.001 *$ \\
\hline GSH & & $120(2.49 \pm 1.002)$ & $120(4.026 \pm 1.479)$ & $<0.001^{*}$ \\
\hline
\end{tabular}

*Significant $P$ value $<0.05$

Table 3. Prevalence of Toxoplasma in schizophrenic patients in relation to gender, residence, pets, ECT, history of drug abuse, family history of psychotic and mood disorders.

\begin{tabular}{|c|c|c|c|c|}
\hline Relative data & & No. of negative (\%) & No. of positive (\%) & $P$ value \\
\hline \multirow[t]{2}{*}{ Gender } & Male & $39(59.1)$ & $42(77.2)$ & \\
\hline & Female & $27(40.9)$ & $12(22.8)$ & $0.03 *$ \\
\hline \multirow{2}{*}{ Residence } & Urban & $45(68.2)$ & $24(44.4)$ & \\
\hline & Rural & $21(31.8)$ & 30 (55.6) & $0.002 *$ \\
\hline \multirow[t]{2}{*}{ Contact with pets } & Negative & 54 (81.8) & 45 (83.3) & \\
\hline & Positive & $12(18.2)$ & $9(16.7)$ & 0.828 \\
\hline \multirow[t]{2}{*}{ ECT } & Negative & $23(34.8)$ & $25(46.3)$ & \\
\hline & Positive & $43(65.2)$ & $29(53.7)$ & 0.202 \\
\hline \multirow[t]{2}{*}{ History of drug abuse } & Negative & $66(100.0)$ & 48 (88.9) & \\
\hline & Positive & $0(0.0)$ & $6(11.1)$ & $0.005^{*}$ \\
\hline \multirow[t]{2}{*}{ Family history of psychosis } & Negative & 54 (81.8) & $33(61.1)$ & \\
\hline & Positive & $12(18.2)$ & $21(38.9)$ & 0.011* \\
\hline \multirow[t]{2}{*}{ Family history of mood disorders } & Negative & 54 (81.8) & 45 (83.3) & \\
\hline & Positive & $12(18.2)$ & $9(16.7)$ & 0.828 \\
\hline
\end{tabular}

$$
\text { *Significant } P \text { value }<0.05
$$

Table 4. PS, NS, GS, TS, Toxoplasma antibody titer, cortisol, free testosterone and GSH levels in relation to Toxoplasma prevalence and gender in schizophrenic patients.

\begin{tabular}{|c|c|c|c|c|c|c|}
\hline & \multirow[b]{2}{*}{ Neg./Pos. } & \multicolumn{2}{|c|}{ Toxplasma } & \multicolumn{3}{|c|}{ Gender } \\
\hline & & No. $($ Mean \pm SD) & $P$ value & Male/Female & No. (Mean \pm SD) & $P$ value \\
\hline \multirow[t]{2}{*}{ PS (7-49) } & Negtive & $66(21.5 \pm 8.6)$ & & Male & $81(22.5 \pm 9.0)$ & \\
\hline & Positive & $54(22.3 \pm 7.9)$ & 0.607 & Female & $39(20.6 \pm 6.53)$ & 0.241 \\
\hline \multirow[t]{2}{*}{ NS (7-49) } & Negtive & $66(18.3 \pm 9.13)$ & & Male & $81(21.11 \pm 9.71)$ & \\
\hline & Positive & $54(19.8 \pm 10.76)$ & 0.403 & Female & $39(14.7 \pm 8.93)$ & 0.001* \\
\hline \multirow[t]{2}{*}{ GS (16-112) } & Negtive & $6626.7 \pm 7.09)$ & & Male & $8126.1 \pm 5.53)$ & \\
\hline & Positive & $54(24.8 \pm 5.04)$ & 0.101 & Female & $39(25.3 \pm 7.69)$ & 0.527 \\
\hline \multirow[t]{2}{*}{ TS } & Negtive & $66(66.6 \pm 16.2)$ & & Male & $81(69.8 \pm 12.9)$ & \\
\hline & Positive & $54(67.1 \pm 12.43)$ & 0.860 & Female & $39(60.6 \pm 15.9)$ & 0.001* \\
\hline \multirow[t]{2}{*}{ Toxoplasma titers } & - & - & & Male & $81(1.27 \pm 1.13)$ & \\
\hline & - & - & - & Female & $39(83.0 \pm 1.06)$ & $0.043^{*}$ \\
\hline \multirow[t]{2}{*}{ Cortisol } & Negtive & $66(163.8 \pm 138.6)$ & & Male & $81(190.3 \pm 135.2)$ & \\
\hline & Positive & $54(193.0 \pm 119.0)$ & 0.223 & Female & $39(149.1 \pm 116.9)$ & 0.105 \\
\hline \multirow[t]{2}{*}{ Males free testosterone } & Negtive & $39(7.0 \pm 6.59)$ & & Male & $81(17.9 \pm 10.48)$ & \\
\hline & Positive & $42(10.8 \pm 6.23)$ & $0.01 *$ & & & \\
\hline \multirow[t]{2}{*}{ Females free testosterone } & Negtive & $27(2.2 \pm 1.65)$ & & Female & $39(6.5 \pm 8.52)$ & 0.001* \\
\hline & Positive & $12(8.5 \pm 9.62)$ & 0.003* & & & \\
\hline \multirow[t]{2}{*}{ GSH } & Negtive & $66(2.8 \pm 1.0)$ & & Male & $81(2.3 \pm 1.01)$ & \\
\hline & Positive & $54(2.0 \pm 0.82)$ & $<0.001^{*}$ & Female & $39(2.7 \pm 0.92)$ & $0.045^{*}$ \\
\hline
\end{tabular}


While free testosterone levels were found to be significantly higher in male patients receiving ECT $(P=0.026)$, no significant difference was found between ECT and PANSS, cortisol and anti-Toxoplasma IgG. Moreover, no significant difference between drug abuse and the previous mentioned study parameters was detected (Table 5). As regards the family history for mood and psychotic disorders, statistical significance was only detected between the family history for mood disorders and the GSH $(P=0.021)$ (Table 6).

Table 5. PS, NS, GS, TS, Toxoplasma antibody titer, cortisol, free testosterone and GSH levels in relation to ECT and history of drug abuse in schizophrenic patients.

\begin{tabular}{|c|c|c|c|c|c|c|}
\hline & \multirow[b]{2}{*}{ Neg./Pos. } & \multicolumn{2}{|l|}{ ECT } & \multicolumn{3}{|c|}{ History of drug abuse } \\
\hline & & No. $($ Mean \pm SD) & $P$ value & Neg./Pos. & No. $($ Mean \pm SD) & $P$ value \\
\hline \multirow[t]{2}{*}{ PS (7-49) } & Negtive & $48(22.0 \pm 5.93)$ & & Negtive & $114(21.71 \pm 8.46)$ & \\
\hline & Positive & $72(21.8 \pm 9.6)$ & 0.915 & Positive & $6(25.5 \pm 2.739)$ & 0.278 \\
\hline \multirow[t]{2}{*}{ NS (7-49) } & Negtive & $48(21.1 \pm 10.45)$ & & Negtive & $114(19.24 \pm 9.92)$ & \\
\hline & Positive & $72(17.6 \pm 9.3)$ & 0.060 & Positive & $6(15.5 \pm 9.311)$ & 0.369 \\
\hline \multirow[t]{2}{*}{ GS (16-112) } & Negtive & $48(26.5 \pm 5.58)$ & & Negtive & $114(25.89 \pm 6.397)$ & \\
\hline & Positive & $72(25.4 \pm 6.73)$ & 0.349 & Positive & $6(26 \pm 4.382)$ & 0.968 \\
\hline \multirow[t]{2}{*}{ TS } & Negtive & $48(69.6 \pm 12.8)$ & & Negtive & $114(66.84 \pm 14.545)$ & \\
\hline & Positive & $72(64.9 \pm 15.43)$ & 0.081 & Positive & $6(67.0 \pm 16.432)$ & 0.979 \\
\hline \multirow[t]{2}{*}{ Toxoplasma titers } & Negtive & $48(1.2 \pm 1.0)$ & & Negtive & $114(1.7371 \pm 1.08)$ & \\
\hline & Positive & $72(1.03 \pm 1.14)$ & 0.251 & Positive & $6(2.385 \pm 0.312)$ & 0.146 \\
\hline \multirow[t]{2}{*}{ Cortisol } & Negtive & $48(161.9 \pm 112.8)$ & & Negtive & $114(174.0921 \pm 128.294)$ & \\
\hline & Positive & $72(187.0 \pm 140.9)$ & 0.284 & Positive & $6(231.7 \pm 172.313)$ & 0.294 \\
\hline \multirow[t]{2}{*}{ Males free testosterone } & Negtive & $27(7.8 \pm 6.69)$ & & - & - & \\
\hline & Positive & $54(11.3 \pm 6.0)$ & $0.026^{*}$ & - & - & - \\
\hline \multirow[t]{2}{*}{ Females free testosterone } & Negtive & $21(4.0 \pm 5.33)$ & & - & - & \\
\hline & Positive & $18(9.5 \pm 10.55)$ & 0.055 & - & - & - \\
\hline \multirow[t]{2}{*}{ GSH } & Negtive & $48(2.3 \pm 0.96)$ & & Negtive & $114(2.5053 \pm 1.009)$ & \\
\hline & Positive & $72(2.5 \pm 1.0)$ & 0.307 & Positive & $6(2.2 \pm .876)$ & 0.469 \\
\hline
\end{tabular}

*Significant $P$ value $<0.05$

Table 6. PS, NS, GS, TS, Toxoplasma antibody titers, cortisol, free testosterone and GSH levels in relation to family history of psychosis and family history of mood disorders in schizophrenic patients.

\begin{tabular}{|c|c|c|c|c|c|c|}
\hline & \multirow[b]{2}{*}{ Neg./Pos. } & \multicolumn{2}{|l|}{ ECT } & \multicolumn{3}{|c|}{ History of drug abuse } \\
\hline & & No. $($ Mean \pm SD) & $P$ value & Neg./Pos. & No. (Mean \pm SD) & $P$ value \\
\hline \multirow[t]{2}{*}{ PS (7-49) } & Negtive & $99(21.33 \pm 7.77)$ & & Negtive & $87(22.17 \pm 8.93)$ & \\
\hline & Positive & $21(24.57 \pm 10.24)$ & 0.105 & Positive & $33(21.18 \pm 6.43)$ & 0.562 \\
\hline \multirow[t]{2}{*}{ NS (7-49) } & Negtive & $99(19.30 \pm 9.55)$ & & Negtive & $87(19.38 \pm 10.21)$ & \\
\hline & Positive & $21(17.86 \pm 11.52)$ & 0.545 & Positive & $33(18.18 \pm 9.05)$ & 0.556 \\
\hline \multirow[t]{2}{*}{ GS (16-112) } & Negtive & $99(25.09 \pm 5.55)$ & & Negtive & $87(26.14 \pm 6.43)$ & \\
\hline & Positive & $72(25.4 \pm 6.73)$ & $0.021 *$ & Positive & $33(25.27 \pm 5.98)$ & 0.504 \\
\hline \multirow[t]{2}{*}{ TS } & Negtive & $21(29.71 \pm 8.15)$ & & Negtive & $87(67.69 \pm 15.4)$ & \\
\hline & Positive & $99(65.73 \pm 14.81)$ & 0.067 & Positive & $33(64.64 \pm 12.04)$ & 0.307 \\
\hline \multirow[t]{2}{*}{ Toxoplasma titers } & Negtive & $21(72.14 \pm 12.31)$ & & Negtive & $87(1.02 \pm 1.11)$ & \\
\hline & Positive & $99(1.08 \pm 1.06)$ & 0.414 & Positive & $33(1.42 \pm 1.15)$ & 0.077 \\
\hline \multirow[t]{2}{*}{ Cortisol } & Negtive & $21(1.35 \pm 1.42)$ & & Negtive & $87(178.25 \pm 136)$ & \\
\hline & Positive & $99(192.28 \pm 104.24)$ & 0.077 & Positive & $33(173.59 \pm 116.74)$ & 0.862 \\
\hline \multirow[t]{2}{*}{ Males free testosterone } & Negtive & $21(243.35 \pm 175.72)$ & & Negative & $60(9.22 \pm 6.47)$ & \\
\hline & Positive & $69(9.18 \pm 6.69)$ & 0.589 & Positive & $21(8.41 \pm 7.24)$ & 0.634 \\
\hline \multirow[t]{2}{*}{ Females free testosterone } & Negtive & $12(8.05 \pm 6.57)$ & & Negative & $27(5.54 \pm 5.26)$ & \\
\hline & Positive & $30(6.57 \pm 8.95)$ & 0.985 & Positive & $12(8.92 \pm 13.29)$ & 0.258 \\
\hline \multirow[t]{2}{*}{ GSH } & Negtive & $99(2.33 \pm 0.94)$ & & Negtive & $87(2.55 \pm 1.01)$ & \\
\hline & Positive & $21(1.98 \pm 0.97)$ & 0.124 & Positive & $33(2.31 \pm 0.98)$ & 0.225 \\
\hline
\end{tabular}




\section{DISCUSSION}

The current study aims to assess the seroprevalence/ serointensity of toxoplasmosis in schizophrenic patients in relation to the levels of free testosterone, cortisol and GSH activity in order to evaluate these factors in schizophrenic patients harboring latent toxoplasmosis. Our results revealing significant higher titers of Toxoplasma in cases group than control group, are assured by other studies ${ }^{[25,26]}$ that reported the risk of schizophrenia in offspring of mothers with maternal antibodies to toxoplasmosis. Accordingly, this appears to implicate the mothers of our cases group in transplacental transmission of toxoplasmosis. Our result also conforms with a meta-analysis that involved forty-two studies carried out in 17 countries over five decades $^{[27]}$. Analysis of meta data that included 3873 individuals with schizophrenia and 7046 controls, revealed that individuals with schizophrenia have an increased prevalence of antibodies to T. gondii. Concepts for this statement include that schizophrenia is known to have a genetic component, and that genes are also known to influence the susceptibility of animals to T. gondii. Schizophrenia is believed to be a disease of neurodevelopment, which is consistent with the known ability of $T$. gondii to cause prenatal infections and remain latent for many years before reactivation. Also while, schizophrenia is known to include abnormalities of neurotransmitters; animal studies have demonstrated an effect of $T$. gondii on dopamine and serotonin. It is also noticed that there are persistent behavioral changes in Toxoplasmainfected animals ${ }^{[27]}$.

Leweke et al. ${ }^{[28]}$ reported that the IgG antibody levels in untreated schizophrenic patients were the highest followed by that in treated patients then controls. This was confirmed in other reports ${ }^{[29,30]}$ in which the prevalence of T. gondii IgG antibodies among patients with schizophrenia was significantly higher than in the controls but not the IgM antibody levels indicating the latency of the infection. Furthermore, other studies ${ }^{[31,32]}$ observed no significant rise in IgG titer and attributed this to the possible effect of antipsychotic medications in causing a reduction of T. gondii IgG level by inhibiting the replication of $T$. gondii. Controversially, the study by Khademvatan et $a l^{[33]}$ indicated differences in Toxoplasma antibody seroprevalence between males and females schizophrenic patients, but not between patients with schizophrenia and healthy individuals nor among different subtypes of schizophrenia, various age groups, residential area, or clinical course of treatment.

In our results Toxoplasma prevalence rate in schizophrenic cases was significantly higher in male patients than in females, and in rural than in urban areas. It was significantly lower, and therefore could not be incriminated, in patients with history of drug abuse and patients with positive family history of psychotic disorders. Prevalence was not higher in patients with history of contact with cats which apparently discounts that issue as a risk factor in these patients. Also, no significant difference was recorded in patients with positive family history of mood disorders, in relation to PS, NS, TS, GSH, treatment with ECT, or level of cortisol. Holub et al. ${ }^{[34]}$ confirmed that higher PANSS scores of positive, negative and disorganized psychopathology were associated with lower titer of anti-Toxoplasma antibodies. In addition, it was postulated that due to the presence of the parasites within neurons, hostmediated neuroimmune and hormonal responses to infection are present ${ }^{[8]}$.

Our results revealed significant higher free testosterone levels (in both genders) and significant higher cortisol levels in cases group than control group. These results conform with the report by Zouei et al. ${ }^{[35]}$ who found that testosterone concentration in toxoplasmosis patients was higher than that in control group with statistically significant difference for men and women. Zghair et al. ${ }^{[36]}$ had previously reported that both acute and chronic toxoplasmosis in males recorded significant higher mean concentration of total and free testosterone. Analysis of our data concerning patients on ECT revealed that free testosterone levels in male patients were significantly higher. However, patients on ECT did not show any other significant relationships with the other parameters. On the other hand, our results were different from those of Eslamirad et al. ${ }^{[17]}$, and Oktenli et $a l^{[37]}$ who demonstrated significantly lower concentration of total and free testosterone in male patients during acute toxoplasmosis. The authors explained this controversy as due to involvement of different parasite strains with variable virulence and epidemiological occurrence; and concluded that the parasite genotype seems to be an important parameter influencing the clinical infection in humans.

GSH is a non-enzymatic antioxidant that eliminates free radicals and has been suggested to have a role in schizophrenia ${ }^{[19]}$. We found that the GSH level was significantly lower in cases group than control group, and in the Toxoplasma positive patients it was significantly lower. Our results conform with those of Karaman et al. ${ }^{[19]}$ and Nucifora et al. ${ }^{[20]}$ who found a statistically significant difference between T. gondiiinfected patients and the control group as regard serum MDA, GSH, and nitric oxide levels. While a decrease in GSH activity was detected, MDA and nitric oxide levels increased significantly; and this decrease in GSH level correlated with PANSS total and positive scores for schizophrenia. In our study gender showed no significance concerning PS, GSH and cortisol levels. While NS, TS and Toxoplasma antibody titers were more significant in male patients than females, GSH level was significantly lower, and family history of mood disorders was found to be significantly related to GSH. Family history of psychotic disorders did not show any significant difference and drug abuse did not show any significant relationship. 
It was reported that behavioral changes associated with latent Toxoplasma infection are attributed to a presumed increase in neurotransmitters signaling ${ }^{[38]}$. Therefore, Toxoplasma may play a role in the etiopathogenesis of psychiatric disorders by affecting neurotransmitters which are implicated in the emergence of psychosis and behavioral abnormalities; and by inducing brain inflammation by the direct stimulation of inflammatory cytokines in the central nervous system ${ }^{[39]}$. Accordingly, neurotransmitterblocking agents have shown some efficacy in the treatment of this pathology. However, the possible association between toxoplasmosis and positive symptoms should be proven by future studies involving neurotransmitters investigations ${ }^{[00]}$. It can therefore be hypothesized that toxoplasmosis is involved in the etiology of schizophrenia at least partially through the elevation of testosterone and the generation of oxidative stress.

Accordingly, it is concluded that in schizophrenic patients, there were high Toxoplasma antibody levels as well as free testosterone and cortisol levels, while there was reduced GSH levels suggesting that latent toxoplasmosis may be involved in the etiology of schizophrenia via neuro-endocrinal alterations. The implication of toxoplasmosis in schizophrenia paves the way to a promising approach to prevention and management of this disease and other psychiatric disorders. This approach adds to the conventional anti-psychotic management strategy by suggesting epidemiological and infectious disease dimension that could provide a background for adjunctive prevention and management plans for patients with schizophrenia.

Author contribution: MSA Abd-El-Tawab suggested research concept. AMR Hamed, NA Mahfouz, SA Hassan and RAE Ibrahim collected samples and data. AM Mahfoz performed ELISA, Elsebaei ES did the statistical analysis, while MK Rehan and NSM El-Gebaly analyzed data. NSM El-Gebaly, MSA Abd-El-Tawab, AMR Hamed and MK Rehan wrote and revised the manuscript.

Competing interest: The authors declare no competing interests.

\section{REFERENCES}

1. Abdoli A, Dalimi A. Are there any relationships between latent Toxoplasma gondii infection, testosterone elevation, and risk of autism spectrum disorder. Front Behav Neurosci 2014; 8: 339-345.

2. Nogareda F, Le Strati Y, Villena I, De Valki H, Goulet V. Incidence and prevalence of Toxoplasma gondii infection in women in France, 1980-2020: Model-based estimation. Epidemiol Infect 2013; 142(8):1-10.

3. Flegr J, Klapilová K, Kaňková S. Toxoplasmosis can be a sexually transmitted infection with serious clinical consequences. Not all routes of infection are created equal; Med Hypoth 2014; 83(3):286289.

4. Dalimi A, Abdoli A. Latent toxoplasmosis and human. Iran J Parasitol 2012; 7(1):1-17.

5. Robert-Gangneux F, Dardé ML. Epidemiology and diagnostic strategies for toxoplasmosis. Clin Microbiol Rev 2012; 25(2):264-296.

6. Hamed AMR, El-Gebaly NSM, Abdelmegeid AK, Elsebaei ES. Seroprevalence of Toxoplasma gondii infection in mentally retarded children in Egypt. PUJ 2018; 11 (3):155-161.

7. Hurley RA, Taber KH. Latent toxoplasmosis gondii: emerging evidence for influences on neuropsychiatric disorders. J Neuropsych Clin Neurosci 2012; 24:4.

8. Flegr J. Influence of latent Toxoplasma infection on human personality, physiology and morphology: pros and cons of the Toxoplasma-human model in studying the manipulation hypothesis. J Exp Biol 2013; 216: 127-133.

9. Tedford E, McConkey G. Neurophysiological changes induced by chronic Toxoplasma gondii infection. J Pathog 2017; 6(2) 19-25.

10. Tandon R, Keshavan M. Schizophrenia NH. Just the facts: what we know in 2008. Schizophr Res 2008; 100:4-9.

11. Yolken RH, Dickerson FB, Torrey FE. Toxoplasma and schizophrenia. Parasit Immunol 2009; 31:706715.

12. Yuksel P, Alpay N, Babur C, Bayar R, Saribas S, Karakose AR, et al. The role of latent toxoplasmosis in the aetiopathogenesis of schizophrenia the risk factor or an indication of a contact with cat? Folia Parasitol (Praha) 2010; 57:121-128.

13. Torrey EF, Bartko JJ, Yolken RH. Toxoplasma gondii and other risk factors for schizophrenia: an update. Schizophr Bull 2012; 38:642-647.

14. Ebrahimzadeh A., Shahraki K, Mohammadi M. Serological and molecular diagnosis of Toxoplasma gondii in patients with schizophrenia. J Parasit Dis. 2018; 42(2): 177-181.

15. Sorlozano-Puerto A, Gutierrez-Fernandez J. Schizophrenia spectrum disorders and their treatments. In: Toxoplasma gondii and schizophrenia: a relationship that is not ruled out. Editor: Shen Y-C. Chapter: 4 2017; 60-93. Croatia http://dx.doi.org/10.5772/66018.

16. Flegr J, Llindova, J, Kodym P. Sex-dependent toxoplasmosis-associated differences in testosterone concentration in humans. Parasitology 2008; 135:427-431.

17. Eslamirad Z, Hajihossein R, Ghorbanzadeh B, Alimohammadi M, Mosayebi M, Didehdar M. Effects of Toxoplasma gondii Infection in level of serum testosterone in males with chronic toxoplasmosis. Iran. J Parasitol 2013; 8:622-626.

18. Ko YH, Jung SW, Joe SH, Lee CH, Jung HG, Jung IK, et al. Association between serum testosterone levels and the severity of negative symptoms in male 
patients with chronic schizophrenia. Psychoneuro Endocrinol 2007; 32(4):385-391.

19. Karaman U, Celik T, Kiran TR, Colak C, Daldal NU. Malondialdehyde, glutathione, and nitric oxide levels in Toxoplasma gondii seropositive patients. Korean J Parasitol 2008; 46(4):293-295.

20. Nucifora LG, Tanaka T, Hayes LN, Kim M, Lee BJ, Matsuda T, et al. Reduction of plasma glutathione in psychosis associated with schizophrenia and bipolar disorder in translational psychiatry. Transl Psychiatry 2017; 7(8):1215-1220.

21. Ventura J, Lieberman R, Green M, Shaner A, Mintz J. Training and quality assurance with the Structured Clinical Interview for DSM-IV (SCID-I/P). Psychiatr Res 1998; 79,163- 173.

22. Kay S, Fiszbein A, Opler L. The positive and negative syndrome scale (PANSS) for schizophrenia. Schizophr Bull 1987; 13: 261.

23. Fallahi E, Badparva M, Mohammadi M, Ebrahimzadeh F, Pournia Y. Sero-epidemiological study of Toxoplasma gondii in women referred to Khorramabad laboratory of health center for medical examination before marriage. Asian J Biol Sci 2009; 2 (3): 88-94.

24. Chan YH, Biostatistics 102: quantitative data-parametric and non-parametric tests. Singapore Med J 2003; 44(8):391-396.

25. Brown AS, Schaefer CA, Quesenberry CP , Liu L, Babulas VP, Susser ES. Maternal exposure to toxoplasmosis and risk of schizophrenia in adult offspring. Am J Psychiatr 2005; 162(4):767-773.

26. Mortensen PB, Nørgaard-Pedersen B, Waltoft BL, Sørensen TL, Hougaard D, Torrey EF, et al. Toxoplasma gondii as a risk factor for early-onset schizophrenia: analysis of filter paper blood samples obtained at birth. Biol Psychiatry 2007; 61(5):688-693.

27. Torrey EF, Bartko JJ, Lun ZR, Yolken RH. Antibodies to Toxoplasma gondii in patients with schizophrenia: a meta-analysis. Schizophr Bull 2007; 33 (3): 729-736.

28. Leweke FM, Gerth CW, Koethe D, Klosterkötter J, Ruslanova I, Krivogorsky B, et al. Antibodies to infectious agents in individuals with recent onset schizophrenia. Eur Arch Psychiatr Clin Neurosci 2004; 254(4):8-35.

29. Park MH, Kwon YJ, Jeong HY, Lee HY, Hwangbo Y, Yoon $\mathrm{HJ}$, et al. Association between intracellular infectious agents and schizophrenia. Clin Psychopharmacol Neurosci 2012; 10(2):117-123.

30. Omar A, Bakar OC, Adam NF, Osman H, Osman A, Suleiman AH, et al. Seropositivity and serointensity of Toxoplasma gondii antibodies and DNA among patients with schizophrenia. Korean J Parasitol 2015; 53(1): 29-34.

31. Jones-Brando L, Torrey EF, Yolken R. Drugs used in the treatment of schizophrenia and bipolar disorder inhibit the replication of Toxoplasma gondii. Schizophr Res 2003; 62: 237-244.

32. Webster JP, Lamberton PHL, Donnelly CA, Torrey EF. Parasites as causative agents of human affective disorders? The impact of antipsychotic, mood-stabilizer and anti-parasite medication on Toxoplasma gondii's ability to alter host behavior. Proc Biol Soc 2006; 273: 1023-1030.

33. Khademvatan S, Khajeddin N, Izadi S, Yousefi E. Investigation of anti-Toxocara and anti-Toxoplasma antibodies in patients with schizophrenia disorder. Schizophr Res Treatment 2014; 2014:230349.

34. Holub D, Flegr J, Dragomirecká E, Rodriguez M, Preiss M, Novák T, et al. Differences in onset of disease and severity of psychopathology between toxoplasmosis-related and toxoplasmosisunrelated schizophrenia. Acta Psychiatr Scand. 2013; 127(3):227-238.

35. Zouei N, Shojaee S, Mohebali M, Keshavarz H. The association of latent toxoplasmosis and level of serum testosterone in humans. BMC Res Notes 2018; (11):365-369.

36. Zghair KH, Al-Qadhi BN, Mahmood SH. The effect of toxoplasmosis on the level of some sex hormones in males blood donors in Baghdad. J Parasit Dis 2015; 39(3):393-400.

37. Oktenli C, Doganci L, Ozgurtas T, Araz RE, Tanyuksel M, Musabak U, et al. Transient hypogonadotrophic hypogonadism in males with acute toxoplasmosis: suppressive effect of interleukin-1 beta on the secretion of GnRH. Hum Reprod 2004; 19(4):859866.

38. Stock AK, Dajkic D, Köhling HL, von Heinegg EH, Fiedler M, Beste C. Humans with latent toxoplasmosis display altered reward modulation of cognitive control. Sci Rep 2017; 7(1): 1017010181.

39. Del Grande C, Galli L, Schiavi E, Dell'Osso L, Bruschi F. Is Toxoplasma gondii a trigger of bipolar disorder? Pathog 2017; 6(1):3-22.

40. Fond G, Macgregor A, Tamouza R, Hamdani N, Meary A, Leboyer $\mathrm{M}$, et al. Comparative analysis of anti-toxoplasmic activity of antipsychotic drugs and valproate. Eur Arch Psychiatr Clin Neurosci 2014; 264(2),179-183. 